\title{
Direct Ultrasonic Aspiration for Orbito-Temporal Neurofibromas
}

\author{
Shinjiro Kono Hirohiko Kakizaki Patricia Ann L. Lee Yasuhiro Takahashi \\ Department of Oculoplastic, Orbital \& Lacrimal Surgery, Aichi Medical University Hospital, \\ Aichi, Japan
}

Keywords

Ultrasonic surgical device · Orbito-temporal neurofibromas · Debulk

\begin{abstract}
Ultrasonic surgical devices are powerful new tools that can debulk soft tissue tumors with little damage to surrounding blood vessels and nerve fibers. We used such a device to remove orbito-temporal neurofibromas, which are usually associated with a rich network of vessels and nerves. Three patients with masses in the upper and lower eyelids and the temporal area of the face were included in this study. The masses were debulked safely using an ultrasonic aspirator, resulting in satisfactory functional and cosmetic outcomes.
\end{abstract}

(c) 2021 The Author(s).

Published by S. Karger AG, Basel

\section{Introduction}

The use of ultrasonic surgical devices in orbital surgeries such as orbital decompressions and biopsy of orbital lesions has gained popularity in recent years [1]. The device works by emulsifying and aspirating bone [1]; moreover, this device selectively fragments and vacuolizes soft tissue with little damage to surrounding blood vessels and nerve fibers [2]. To our knowledge, this device has not been used for the removal of orbito-temporal neurofibromas [3].

Surgical removal of orbito-temporal neurofibromas is challenging [4]. These tumors grow in a narrow orbito-temporal space, infiltrate surrounding tissues, and form rich vascular networks $[4,5]$. Surgeons have conventionally excised neurofibromas with scissors and forceps [6]; however, this procedure has a high risk of tissue damage and hemorrhage [4, 5]. Herein, we demonstrate the benefits and safety of using an ultrasonic surgical device in the removal of orbito-temporal neurofibromas in 3 patients. 
Kono et al.: Ultrasonic Aspiration for Orbito-Temporal Neurofibromas

\section{Report of Cases}

Three patients ( 2 males and 1 female; aged 9, 18, and 26 years) were included in this case series. Tumor removal was done via eyelid crease, swinging eyelid, and suprabrow-to-temporal approaches, respectively. One patient also had a concomitant upper eyelid epiblepharon while the other 2 patients had upper eyelid blepharoptosis. All patients were followed up for at least 6 months.

All surgeries were performed under general anesthesia with local anesthetic injection $(1 \%$ lidocaine and epinephrine diluted to $1: 100,000$ ) by one of the authors (H.K.). Following skin incision, neurofibromas were identified and debulked with an ultrasonic surgical device (SONOPET UST-2001; Stryker Japan K.K.; Tokyo, Japan) fitted with a soft tissue tip with an outside diameter of $1.9 \mathrm{~mm}$. The ultrasonic power level, suction level, and irrigation rate were set at $70 \%, 50 \%$, and $10 \mathrm{~mL} / \mathrm{min}$, respectively. Debulking continued until normal tissues were identified. No significant intraoperative bleeding was noted. The Hotz procedure was also performed in the patient with epiblepharon while levator advancement was performed in the other 2 patients. None of the patients developed postoperative hematomas. All patients showed satisfactory functional and cosmetic outcomes. We present one representative patient below.

\section{Case Presentation}

An 18-year-old male, previously diagnosed case of von Recklinghausen disease, presented to the clinic with a right-sided orbital mass over the temporal half of his face with involvement of both upper and lower eyelids and conjunctivae (Fig. 1a).

Excision biopsy was planned with a working diagnosis of neurofibroma. A suprabrow-totemporal approach was utilized by creating an incision along a previous scar on the lateral forehead. Using an ultrasonic surgical device, the tumor was debulked as much as possible with little bleeding and damage to surrounding tissues (Fig. 1b, c). A lateral canthotomy was also done to remove the conjunctival component of the mass. The Hotz procedure was performed for correction of the upper eyelid epiblepharon.

Six months after the surgery, eyelid opening and closing and ocular motility were within normal range. Cosmesis was generally improved (Fig. 1d).

\section{Discussion}

Ultrasonic surgical devices have previously been used to debulk firm orbital tumors associated with immunoglobulin G4 ophthalmic disease, lacrimal sac adenocarcinoma, and granulomatosis with polyangiitis [3]. Our experience shows, however, that the use of ultrasonic surgical devices for softer, more friable tumors such as orbital neurofibromas is both effective and safe. The soft tissue aspiration system avoids inflicting damage on surrounding vessels and nerves while the irrigation system provides surgeons with a clear surgical field and subsequently shorter surgery times. A maneuverable foot pedal allows surgeons to respond quickly to various intraoperative situations. The multifunctional handpiece also leaves one hand free for the use of other instruments. These provide unique advantages over conventional tools, such as scissors and forceps. Some disadvantages associated with the use of an ultrasonic aspirator include a greater risk for thermal damage to surrounding tissues, increased difficulty aspirating widely spread tumors, and its high cost [1].

In conclusion, our experience demonstrates that using an ultrasonic surgical device to remove neurofibromas is both effective and safe. Its application in the removal of other soft tissue tumors holds great promise.

\section{Karger'}



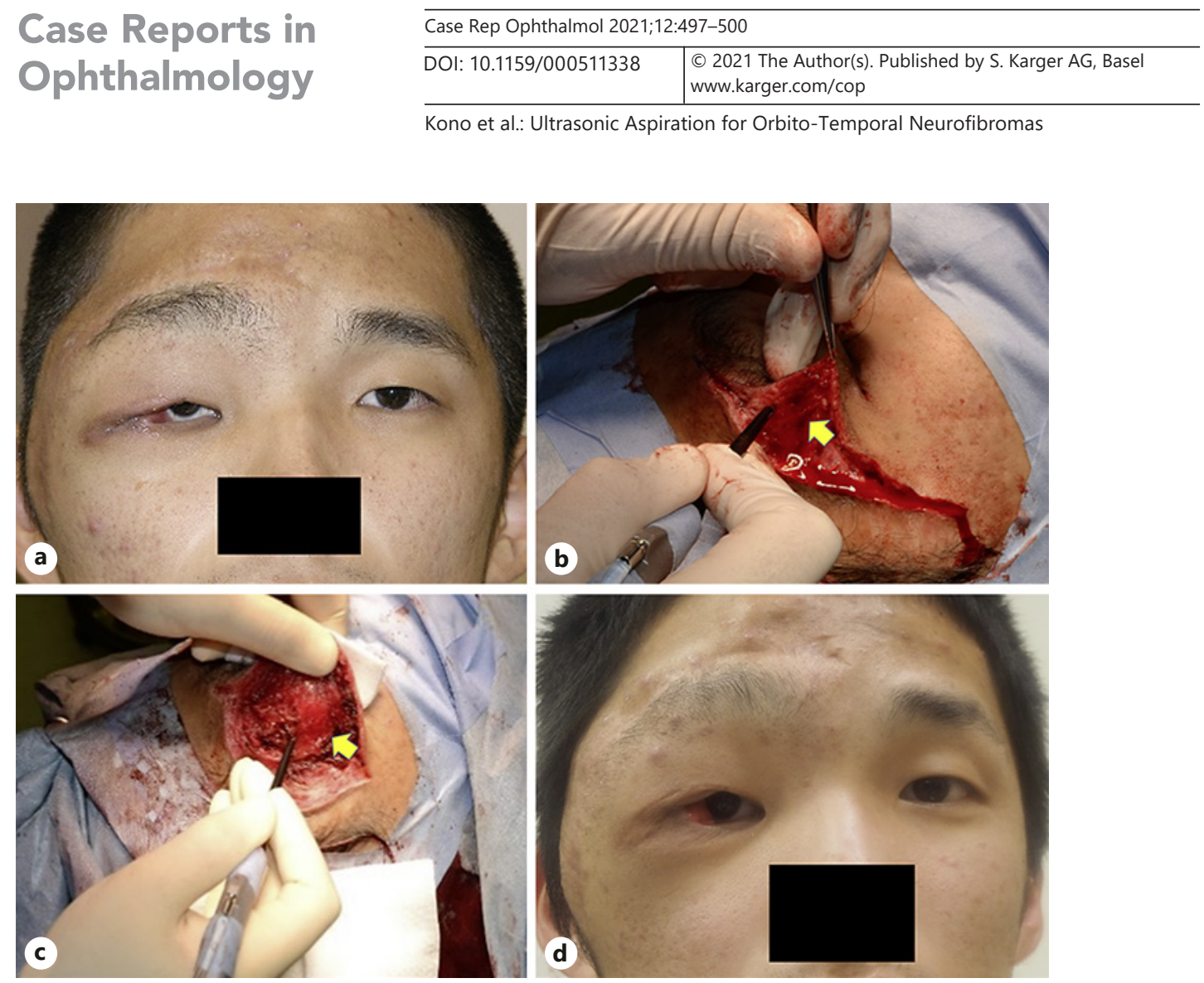

Fig. 1. Case presentation. a Face photos taken before the surgery from the front. A mass is located in the orbito-temporal area of the face and occupies the eyelid and the conjunctiva in part. $\mathbf{b}, \mathbf{c}$ The subcutaneous (b) and main lesions of the tumor (c) were removed using an ultrasonic surgical device (arrows). d Face photos taken 6 months after the surgery from the front.

\section{Statement of Ethics}

This study was conducted in accordance with the tenets of the Declaration of Helsinki and its later amendments. Written informed consent for publication of face photos was obtained from the father of the patient reported in the "Case Presentation" section.

\section{Conflict of Interest Statement}

The authors declare that there is no conflict of interest.

\section{Funding Sources}

The work received no funding.

\section{Author Contributions}

All authors qualify for authorship based on contributions to the conception and design (H.K.), acquisition of data (S.K., H.K., and Y.T.), literature search (S.K. and Y.T.), and interpretation of data (all authors). All authors contributed to drafting the article and revising it critically for important intellectual content and final approval of the version to be published. 


\section{References}

1 Vrcek I, Starks V, Mancini R, Gilliland G. Use of an ultrasonic bone curette (Sonopet) in orbital and oculoplastic surgery. Proc (Bayl Univ Med Cent). 2015 Jan;28(1):91-3.

2 Henzi S, Krayenbühl N, Bozinov O, Regli L, Stienen MN. Ultrasonic aspiration in neurosurgery: comparative analysis of complications and outcome for three commonly used models. Acta Neurochir. 2019 Oct;161(10): 2073-82.

3 Sun SY, Stewart K, Lyford-Pike S, Garrity JA, Harrison AR, Mokhtarzadeh A. Ultrasonic aspiration for debulking infiltrative masses of the orbit. Ophthalmic Plast Reconstr Surg. 2020 Mar-Apr;36(2):198-201.

4 Altan-Yaycioglu R, Hintschich C. Clinical features and surgical management of orbitotemporal neurofibromatosis: a retrospective interventional case series. Orbit. 2010 Oct;29(5):232-8.

5 Lee V, Ragge NK, Collin JR. Orbitotemporal neurofibromatosis. Clinical features and surgical management. Ophthalmology. 2004 Feb;111(2):382-8.

6 Singhal D, Chen YC, Chen YR, Chen PK, Tsai YJ. Soft tissue management of orbitotemporal neurofibromatosis. J Craniofac Surg. 2013 Jan;24(1):269-72. 